\title{
Conexões
}

Educação Física, Esporte e Saúde

DOI 10.20396/conex.v17i0.8655148

Artigo Original

\section{Os efeitos da idade e do sexo no nível de ansiedade de nadadores de águas abertas}

\author{
Ivan Wallan Tertuliano ${ }^{1}$ \\ Fernanda Karen Buzeto ${ }^{1}$ \\ Vivian de Oliveira ${ }^{2}$ \\ Afonso Antonio Machado ${ }^{3}$ \\ José Maria Montiel ${ }^{4}$
}

\section{RESUMO}

Objetivo: O presente estudo teve como objetivo investigar a ansiedade pré-competitiva em nadadores de águas abertas e analisar se a idade e o sexo alteram os níveis de ansiedade pré-competitiva. Metodologia: Para isso, o estudo contou com a participação voluntária de 129 nadadores com idade entre 14 e 49 anos de idade, de ambos os sexos, todos participantes de competições de águas abertas. Os participantes responderam um questionário. O questionário adotado foi o CSAI-2R (Competitive State Anxiety Inventory - 2r, versão reduzida). Resultados e discussão: Os resultados demonstraram que a autoconfiança apresentou valores elevados para todos os grupos amostrais e que a idade e o sexo não são fatores determinantes para os resultados. Além disso, foi encontrada uma correlação forte entre a subescala somática e a cognitiva, corroborando a teoria Multidimensional da ansiedade, na qual diz que quando a autoconfiança apresenta escores elevados, a ansiedade é baixa. Conclusão: Diante disso, os resultados mostraram que independentemente da idade e do sexo, os nadadores de águas abertas apresentam valores elevados de autoconfiança, o que influencia os escores baixos de ansiedade cognitiva e somática.

Palavras-chave: Ansiedade. Natação. Psicologia do Esporte. CSAI-2R.

${ }^{1}$ Centro Universitário Adventista de São Paulo (UNASP), Campus São Paulo, Departamento de Educação Física, São Paulo - SP, Brasil.

2 Universidade Estadual Paulista (UNESP), Instituto de Biociências, Departamento de Educação Física, Campus Rio Claro, Rio Claro - SP, Brasil.

3 Centro Universitário FIEO (UNIFIEO), Departamento de Educação Física, Osasco - SP, Brasil.

4 Universidade São Judas Tadeu (USJT), Departamento de Psicologia, São Paulo - SP, Brasil.

\section{Correspondência:}

Ivan Wallan Tertuliano. Centro Universitário Adventista de São Paulo (UNASP), Departamento de Educação Física, Campus São Paulo, Estrada de Itapecerica, 5859, CEP 05858001, São Paulo - SP, E-mail: ivanwallan@gmail.com

Recebido em: 8 abr. 2019

Aprovado em: 16 ago. 2019 


\section{The effects of age and sex in the anxiety level of open- water swimmers}

\section{ABSTRACT}

Objective: The present study aimed to investigate pre-competitive anxiety in open-water swimmers and to analyze whether age and sex alter pre-competitive anxiety levels. Method: For this, the study counted on the voluntary participation of 129 swimmers between 14 and 49 years of age, of both sexes, all participants of competitions of open waters. Participants answered a questionnaire. The questionnaire adopted was CSAI-2R (Competitive State Anxiety Inventory - 2nd, reduced version). Results and discussion: The results showed that self-confidence presented high values for all sample groups and that age and sex were not determinant factors for the results. In addition, a strong correlation was found between the somatic and cognitive subscales, corroborating the Multidimensional anxiety theory, in which he says that when self-confidence has high scores, anxiety is low. Conclusion: Therefore, the results showed that, regardless of age and sex, open water swimmers have high values of self-confidence, which influences the low scores of cognitive and somatic anxiety.

Keywords: Anxiety. Swimming. Sports Psychology. CSAI-2R.

\section{Los efectos de la edad y del sexo en el nivel de ansiedad de nadadores de aguas abiertas}

\section{RESUMEN}

Objetivo: El presente estudio tuvo como objetivo investigar la ansiedad precompetitiva en nadadores de aguas abiertas y analizar si la edad y el sexo alteran los niveles de ansiedad precompetitiva. Metodología: Para ello, el estudio contó con la participación voluntaria de 129 nadadores con edad entre 14 y 49 años, de ambos sexos, todos participantes de competiciones de aguas abiertas. Los participantes respondieron un cuestionario. El cuestionario adoptado fue el CSAI-2R (Competitive State Anxiety Inventory - 2nd, versión reducida). Resultados y Discusión: Los resultados mostraron que la autoconfianza presentaba valores altos para todos los grupos de la muestra y que la edad y el sexo no eran factores determinantes para los resultados. Además, se encontró una fuerte correlación entre las subescalas somática y cognitiva, corroborando la teoría de la ansiedad multidimensional, en el que dice que cuando la confianza en sí mismo tiene puntuaciones altas, la ansiedad es baja. Conclusión: Por lo tanto, los resultados mostraron que, independientemente de la edad y el sexo, los nadadores de aguas abiertas tienen valores altos de confianza en sí mismos, lo que influye en las puntuaciones bajas de la ansiedad cognitiva y somática.

Palabras Clave: Ansiedad. Natación. Psicología del Deporte. CSAI-2R. 


\section{INTRODUÇÃO}

Durante a competição os atletas devem dar conta de situações que podem gerar ansiedade e alguns estudos apontam que a ansiedade pode ser um fator que influencia os resultados dos atletas, muitas vezes de forma negativa (MILAVIĆ; JURKO; GRGANTOV, 2013; MOLINA; SANDÍN; CHOROT, 2014; NOGUEIRA; BARA FILHO; LOURENÇO, 2015). Ansiedade pode ser definida como aflição, angústia, perturbação do espírito causadas pela incerteza do perigo (GLEITMAN; REISBERG; GROSS, 2009).

Todavia, cabe tecer que os níveis de ansiedade são individuais, ou seja, as pessoas apresentam níveis diferentes de ansiedade e mesmo assim alcançam os mesmos resultados nas tarefas. Assim, para a maioria das pessoas, um grau elevado de ansiedade pode prejudicar o desempenho (WEINBERG; GOULD, 2017). Diante do exposto, ansiedade é vista como uma das principais variáveis que interferem no desempenho dos atletas (ARRUDA et al., 2017; FORTES et al., 2017; HAGAN JR.; POLLMANN; SCHACK, 2017; BAUZÁ et al., 2018; SONOO et al., 2010).

Ansiedade é um construto multidimensional que se refere à disposição para responder ao estresse e à tendência de perceber situações estressantes (FERNANDES et al., 2013a). Samulski (2009) aponta que o principal estressor para os atletas é a competição. A competição de natação, em particular, tem algumas características específicas que podem aumentar a ansiedade competitiva. $O$ contato visual do atleta com seu oponente momentos antes da competição e a possibilidade de ouvir o locutor pronunciando seu nome e o de seus oponentes momentos antes de entrar na água para competir podem potencializar a ansiedade competitiva.

Diferentes instrumentos foram desenvolvidos, com o intuito de compreender melhor o papel da ansiedade no rendimento dos atletas, como o STAI (State-trait anxiety inventory) que no Brasil é conhecido como IDATE - inventário de Ansiedade traço-estado (BIAGGIO; NATALÍCIO; SPIELBERGER, 1977) e o CSAI-2 (Competitive State Anxiety Inventory - 2) (MARTENS; VEALEY; BURTON, 1990). Desses instrumentos, o CSAI-2 foi o instrumento escolhido no presente estudo pois parte do pressuposto de mensurar ansiedade a partir de três subescalas: ansiedade de estado cognitiva (expectativas negativas quanto ao desempenho), ansiedade de estado somática (sensações físicas, sintomas propriamente ditos de ansiedade) e autoconfiança (ausência de ansiedade, sentimento positivos quanto ao desempenho) (BAUZÁ et al., 2018), o que considera-se relevante para o constructo multidimensional adotado neste manuscrito.

Utilizando-se do CSAI-2, alguns estudos foram conduzidos, com o objetivo de identificar a ansiedade de traço e a ansiedade de estado em atletas no esporte (BOAS et al., 2012; FERNANDES et al., 2013a; PALUDO et al., 2016; SONOO et 
al., 2010; SOUZA; TEIXEIRA; LOBATO, 2012; VIEIRA et al., 2011). No Brasil o instrumento CSAI-2 foi validado para língua portuguesa (COELHO; VASCONCELOS-RAPOSO; MAHL, 2010), bem como sua versão reduzida, o CSAI2R (Competitive State Anxiety Inventory - 2R, versão reduzida) (COX; MARTENS; RUSSELL, 2003), a qual foi validade e traduzida por Fernandes et al. (FERNANDES; VASCONCELOS-RAPOSO; FERNANDES, 2012a). O CSAI-2R contém menos itens que o original (17 itens), porém com a mesma qualidade psicométrica (FERNANDES et al., 2014; PALUDO et al., 2016).

Paludo et al. (2016) realizaram uma ampla revisão da literatura acerca da relação entre ansiedade e desempenho esportivo, avaliada pelo CSAI-2 e que aplicaram análise fatorial confirmatória para verificar as propriedades psicométricas do instrumento, demonstrando que o CSAI-2 apresentou propriedades psicométricas consideráveis em diferentes contextos, inclusive para sua versão reduzida, o CSAI-2R (FERNANDES; VASCONCELOS-RAPOSO; FERNANDES, 2012a). Somado a isso, outros estudos tem apontado consistência nos índices de ajustamento da versão reduzida (CSAI-2R) para avaliação da ansiedade pré-competitiva (COX; MARTENS; RUSSELL, 2003; FERNANDES et al., 2014; FERNANDES; VASCONCELOS-RAPOSO; FERNANDES, 2012a; BAUZÁ; VILADRICH; RAMIS, 2017).

Além disso, o CSAI-2R, por partir de um constructo multidimensional, avalia 3 diferentes dimensões da ansiedade (intensidade, direção e frequência). Intensidade diz respeito aos níveis (intensidade) dos sintomas cognitivos e somáticos (HAGAN JR.; POLLMANN; SCHACK, 2017). A direção é descrita como interpretação dos atletas da intensidade dos sintomas cognitivos e somáticos como positivo ou negativo em relação ao desempenho subsequente (JONES; SWAIN, 1992), enquanto que a frequência é definida como a quantidade de tempo que um atleta passa atendendo aos sintomas experimentados em relação à competição (SWAIN; JONES, 1993).

A intensidade é avaliada em uma escala Likert de 4 pontos variando de 1 (nada) a 4 (muito). A dimensão direção é avaliada numa escala Likert bipolar de 7 pontos variando de -3 (muito debilitante / negativa) a +3 (muito facilitadora / positiva), de acordo com a percepção de sintomas debilitantes ou facilitadores, com 0 indicando que o atleta percebeu que o sintoma não era importante. Por fim, a dimensão de frequência é avaliada em uma escala Likert de 7 pontos, ancorada por 1 (nunca) e 7 (muito frequentemente) (JONES, 1995).

Retornando, de acordo com Englert e Bertrams (2012) o efeito da ansiedade ainda é um fator a ser investigado no meio esportivo e, mais do que isso, segundo Keisha, Yusof e Jourskesh (2015), os estudos atuam, em sua maioria, em esportes coletivos, o que demonstra necessidade de estudos no âmbito dos esportes individuais, como por exemplo na natação, justificando o presente estudo. Assim, o objetivo do presente estudo é investigar a ansiedade pré-competitiva em 
nadadores de águas abertas e analisar se a idade e o sexo alteram os níveis de ansiedade pré-competitiva.

\section{MÉTODO}

\section{PARTICIPANTES}

O estudo contou com a participação voluntária de 129 pessoas, sendo 49 do sexo feminino ( $38 \%$ ) e 80 do sexo masculino (62\%), todos nadadores de águas abertas de clubes e academias do Estado de São Paulo, com idade entre 14 e 49 anos ( $M=20,28, D P=8,07)$. Os nadadores foram divididos em 3 grupos, adotando critério de divisão por idade, que é o mesmo critério que a Confederação Brasileira de Desporto Aquática (CBDA) adota para divisão das categorias. Cabe destacar que a abrangência relativa à faixa etária dos participantes deste estudo foi devido à possibilidade de averiguação de diferenças nos resultados obtidos no instrumento utilizado, e que foi objetivo deste estudo. Sendo assim, os grupos formados foram: Grupo 1 - 36 nadadores com idade entre 14 e 16 anos (categorias infantil II, juvenil I e juvenil II); Grupo 2 - 36 nadadores com idade entre 17 e 19 anos (categorias júnior I e II); Grupo 3 - 57 nadadores com idade entre 20 e 49 anos (categorias sênior e master).

Na segunda análise, comparou-se homens e mulheres, formando dois grupos amostrais: Grupo masculino, formado por 80 homens e grupo feminino, formado por 49 mulheres. Na tabela 1 são apresentadas as características gerais da amostra do estudo.

\begin{tabular}{cc} 
Tabela 1 - Características gerais da amostra do estudo $(\mathrm{n}=129)$ \\
\hline Variáveis & $\mathbf{n}(\mathbf{\%})$ \\
\hline Sexo & $49(38 \%)$ \\
Feminino & $80(62 \%)$ \\
Masculino & $36(27,90 \%)$ \\
Grupos & $36(27,90 \%)$ \\
Grupo $1(14$ a 16 anos) & $57(44,20 \%)$ \\
Grupo $2(17$ a 19 anos) & $M \pm D P$ \\
Grupo $3(20$ a 49 anos) & $20,28 \pm 8,07$ \\
\hline
\end{tabular}

Todos os participantes preencheram e assinaram o termo de consentimento livre e esclarecido (TCLE) e, no caso dos menores, além do TCLE, o termo de assentimento. Além disso, cabe ressaltar que o uso de adolescentes no presente estudo é suportado pelo estudo de Bartholomeu, Montiel e Machado (2013), o qual demonstrou adequada estrutura interna para o CSAI-2, mesmo aplicado em adolescentes a partir dos 14 anos e pelo estudo de Vieira et al. (2011), que utilizou 
atletas de 14 a 19 anos para avaliar ansiedade em atletas de atletismo.

\section{INSTRUMENTOS}

Utilizou-se o questionário de informação sociodemográfica, com informações sobre sexo, idade e clube que pertenciam. Além disso, os participantes responderam a versão brasileira reduzida do CSAI-2, denominada de CSAI-2R (FERNANDES; VASCONCELOS-RAPOSO; FERNANDES, 2012a), baseada no CSAI2 (Competitive State Anxiety Inventory - 2) (MARTENS; VEALEY; BURTON, 1990). Este é um instrumento constituído por 17 itens, que medem três subescalas: ansiedade cognitiva (itens 2, 5, 8, 11 e 14), ansiedade somática (itens 1, 4, 6, 9, 12,15 e 17) e autoconfiança (itens 3, 7, 10, 13 e 16) na dimensão intensidade, ou seja, o instrumento parte do constructo multidimensional proposto por Martens, Vealey e Burton (1990). Os itens foram respondidos de acordo com uma escala do tipo Likert de quatro pontos, variando de 1 (nada) a 4 (muito).

Para calcular o escore de cada subescala deve-se somar as respostas dos itens de cada subescala e dividir pelo respectivo número de itens, podendo variar, o valor, entre 1 e 4. Assim, no presente estudo, a dimensão intensidade foi investigada, assim como no estudo de validação da versão de 17 itens do CSAI2R (FERNANDES; VASCONCELOS-RAPOSO; FERNANDES, 2012a).

\section{Procedimentos}

O presente estudo respeitou os princípios legais de uma pesquisa que envolve coleta de dados com seres humanos, ou seja, utilizou-se de etapas estabelecidas pela comunidade científica e respeitou-se todos os padrões éticos de pesquisas com seres humanos do Conselho Nacional de Saúde (CNS), Resolução 466/12. O presente trabalho foi apresentado ao Comitê de Ética e Pesquisa (CEP) do Hospital e Centro de Reabilitação da AACD e aprovado pelo mesmo comitê, sob o número de parecer: 1.541.273.

Após a devida autorização do CEP para iniciar a pesquisa, buscou-se contato com os técnicos para autorizarem a coleta de dados com seus nadadores. Com a devida autorização dos técnicos, os atletas foram informados dos objetivos do estudo e assinaram um termo de consentimento livre e esclarecido (TCLE), garantindo-se o anonimato e confiabilidade de todas as informações coletadas. Para os nadadores menores de 18 anos, o TCLE foi assinado por um responsável maior de idade e o menor assinou o termo de assentimento.

Respeitando o objetivo do estudo, alguns cuidados metodológicos foram adotados, para garantir a confiabilidade das informações coletas, de acordo com as previsões teóricas acerca da ansiedade competitiva. Para isso, os nadadores foram orientados a responder o questionário entre 2 horas e 30 minutos antes da competição, com a preocupação de que os questionários fossem respondidos em 
contexto pré-competitivo e não em contexto de treino.

Os nadadores responderam o questionário individualmente, na presença de um pesquisador responsável e só tinham contato com o questionário no momento do preenchimento, o qual durou aproximadamente 10 minutos. Assim não houve a troca de informações pelos nadadores antes e durante a aplicação do questionário. Após todos nadadores responderem ao questionário, as informações coletadas foram transferidas para uma planilha eletrônica (Excel, versão 2016), para melhor controle e posterior análise dos resultados.

\section{ANÁLISE ESTATÍSTICA}

Para análise dos dados utilizou-se inicialmente a estatística descritiva (média e desvio padrão) para cada subescala do instrumento. Além disso, para verificar a assimetria da distribuição foram utilizados os coeficientes assimetria e curtose, sendo considerados valores aceitáveis os que se encontravam entre -1 e +1 . Analisou-se, também a homogeneidade de variância através do Levene.

Frente a confiabilidade das subescalas do instrumento, utilizou-se o alpha de Cronbach (a de Cronbach), assumindo valores entre 0,7 e 0,8 como aceitáveis (FIELD, 2009). A associação entre variáveis foi conduzida através do coeficiente de correlação de Pearson. Os valores de correlação foram avaliados conforme proposto por Hopkins (2002), em que <0,10 (trivial), 0,10 a 0,30 (baixa), 0,31 a 0,50 (moderada), 0,51 a 0,70 (alta), 0,71 a 0,90 (muito alta), 0,91 a 0,99 (quase perfeita) e 1 (perfeita).

Dessa forma, após a especificação dos índices de normalidade, homogeneidade de variância e consistência interna das subescalas do instrumento, analisou-se diferenças entre as subescalas, utilizando todos participantes, através do uso da Anova one-way, com post-hoc de Bonferroni. Para controle do erro do tipo 1, no Post Hoc de Bonferroni houve a divisão do $\mathrm{p}$ pelo número de comparações (GREEN; SALKIND; AKEY, 2000). Dando continuidade as análises, avaliou-se a diferença entre as subescalas separando a amostra por idade e sexo.

A análise entre grupos de idade foi conduzida com o uso da Anova one-way, com post-hoc de Bonferroni e a análise entre grupos de sexo foi realizada com o uso do Teste $t$ independente. Para analisar diferenças intragrupo utilizou-se de Anova one-way e post-hoc de Bonferroni. Todas as análises estatísticas foram realizadas usando-se o IBM SPSS Statistics, versão 20 e assumiu-se o nível de significância de $p<0,05$.

\section{RESULTADOS E DisCUSSÃO}

Para as análises de normalidade, ao analisar os coeficientes de assimetria e 
curtose, observou-se que todos ficaram entre -1 e +1 , o que dá indícios de que não houve violação no pressuposto de normalidade (KLINE, 2010). Somado a isso, a análise de homogeneidade de variância, com o teste de Levene, demonstrou homogeneidade de variância $(p>0,05)$ para todos os grupos de idade e sexo.

Dessa forma, os dados desse estudo apresentam indícios de uma distribuição normal e homogeneidade de variância, podendo ser avaliados por testes paramétricos. Em relação à consistência interna das subescalas (ansiedade cognitiva, ansiedade somática e autoconfiança), confiabilidade das escalas, o alpha de Cronbach revelou bons índices de confiabilidade ( $a>0,752$ ), e a correlação item-subescala apresentou valores que variaram entre 0,463 e 0,805, ou seja, o instrumento apresenta consistência interna e confiabilidade nas subescalas adotadas, além de uma boa correlação entre os itens e a subescala dos mesmos (variação entre 0,51 e 0,84), independentemente da separação por idade ou de sexo. Esses valores de correlação indicam que as correlações variaram de moderada a muito alta (HOPKINS, 2002).

Frente a análise com todos participantes, para as 3 subescalas do instrumento (somática, cognitiva e autoconfiança), os resultados apresentaram diferenças significantes entre as subescalas $[F(1,67 ; 213,88)=54,063 ; p<0,001]$. O Post hoc apontou para diferenças entre a subescala autoconfiança e as demais $(p<0,025$, valor de $p$ ajustado), ou seja, a autoconfiança dos nadadores, deste estudo, apresentou valores superiores às demais subescalas. Em relação ao sexo, os resultados não apresentaram diferenças significantes para nenhuma subescala $(p>0,05)$, ou seja, homens e mulheres apresentam resultados similares.

Em relação às análises entre grupos de idade, os resultados demonstraram diferença significante na subescala somática $(p<0,05)$ (Tabela 2$)$. O Post hoc demonstrou que a diferença foi entre o grupo 1 e o grupo 3 (valor encontrado foi de $p=0,003$, $p$ adotado para significância foi de $p<0,025$, valor de $p$ ajustado em função da correção de Bonferroni para o post hoc), apontando um valor de ansiedade somática maior no grupo 3, comparado com o grupo 1 . Nas demais subescalas (cognitiva e autoconfiança) não houve diferenças significantes entre grupos, ou seja, os grupos apresentam escores similares.

Tabela 2 - Análise entre grupos para cada subescala $(n=129)$

\begin{tabular}{ccccc}
\hline & & $\begin{array}{c}\text { Ansiedade } \\
\text { Cognitiva }\end{array}$ & $\begin{array}{c}\text { Ansiedade } \\
\text { Somática }\end{array}$ & Autoconfiança \\
\hline Grupo 1 & $\mathrm{M} \pm \mathrm{SD}$ & $1,83 \pm 0,77$ & $1,64 \pm 0,45$ & $2,61 \pm 0,77$ \\
Grupo 2 & $\mathrm{M} \pm \mathrm{SD}$ & $1,97 \pm 0,68$ & $1,72 \pm 0,47$ & $2,43 \pm 0,67$ \\
Grupo 3 & $\mathrm{M} \pm \mathrm{SD}$ & $1,98 \pm 0,57$ & $1,98 \pm 0,585$ & $2,51 \pm 0,58$ \\
\hline & $\mathrm{F}$ & 0,616 & 5,581 & 0,646 \\
& $\mathrm{p}$ & 0,542 & $0,005^{*}$ & 0,526 \\
\hline
\end{tabular}

* diferença significante entre o grupo 1 e o grupo 3, com valores superiores no grupo 3. 
Nas comparações intragrupo (Tabela 3), os resultados apresentaram diferença significante para todos os grupos, com $p<0,05$. Em relação ao grupo 1 , frente a localização da diferença, os resultados demonstraram que a diferença foi entre a subescala autoconfiança e as demais (valor encontrado foi de $p=0,001, p$ adotado para significância foi de $p<0,025$, valor de $p$ ajustado em função da correção de Bonferroni para o post hoc), ou seja, o grupo 1 apresentou escores de autoconfiança superiores aos de ansiedade somática e cognitiva.

Seguindo, para o grupo 2, o post hoc apresentou diferença significante entre as subescalas autoconfiança e ansiedade somática (valor encontrado foi de $p=0,015, p$ adotado para significância foi de $p<0,025$, valor de $p$ ajustado em função da correção de Bonferroni para o post hoc), ou seja, o grupo 2 apresentou escores de autoconfiança superiores aos de ansiedade somática.

Por fim, para o grupo 3 os resultados apresentaram diferença significante entre a subescala autoconfiança e as demais (valor encontrado foi de $p=0,001, p$ adotado para significância foi de $p<0,025$, valor de $p$ ajustado em função da correção de Bonferroni para o post hoc), ou seja, o grupo 3 apresentou escores de autoconfiança superiores aos de ansiedade somática e cognitiva, características similares ao grupo 1.

Tabela 3 - Análise intragrupo para cada subescala $(n=129)$

\begin{tabular}{|c|c|c|c|}
\hline & $\begin{array}{l}\text { Grupo } 1 \\
(n=36) \\
M \pm S D\end{array}$ & $\begin{array}{l}\text { Grupo } 2 \\
(n=36) \\
M \pm S D\end{array}$ & $\begin{array}{l}\text { Grupo } 3 \\
(n=57) \\
M \pm S D\end{array}$ \\
\hline Ansiedade Cognitiva & $1,83 \pm 0,77$ & $1,98 \pm 0,68$ & $1,98 \pm 0,56$ \\
\hline Ansiedade Somática & $1,64 \pm 0,45$ & $1,72 \pm 0,47$ & $1,98 \pm 0,58$ \\
\hline Autoconfiança & $2,61 \pm 0,77$ & $2,44 \pm 0,67$ & $2,52 \pm 0,57$ \\
\hline$F$ & 19,804 & 13,974 & 22,588 \\
\hline $\mathrm{p}$ & $0,001 *$ & $0,001 * *$ & $0,001^{*}$ \\
\hline
\end{tabular}

* diferença significante entre autoconfiança e as demais subescalas

** diferença significante entre autoconfiança e ansiedade somática

Em relação à associação entre as subescalas (Tabela 4), a Correlação de Pearson demonstrou que, frente a todos os participantes, houve correlação moderada entre as subescalas somática e cognitiva $(r=0,43, p<0,001)$, ou seja, quando uma das subescalas aumenta o escore, ocorre o mesmo com a outra. Para as correlações intragrupo os resultados demonstraram valores moderados para ansiedade somática e cognitiva para os grupos $1(r=0,30, p<0,05)$ e $3(r=0,49$, $\mathrm{p}<0,05)$. Todavia, o grupo 2 apresentou correlação elevada para ansiedade somática e cognitiva $(r=0,52, p<0,05)$. Tais valores sugerem que quando uma das subescalas aumenta o escore, ocorre o mesmo com a outra, independentemente de se avaliar a amostra total ou separá-la por idade. 
Tabela 4 - Análise de correlação de Pearson $(n=129)$

\begin{tabular}{|c|c|c|c|}
\hline & & $\begin{array}{c}\text { Ansiedade } \\
\text { Somática } \\
\text { r(p) }\end{array}$ & $\begin{array}{c}\text { Autoconfiança } \\
r(p)\end{array}$ \\
\hline \multirow[b]{2}{*}{ Todos } & Ansiedade & $\begin{array}{c}0,431 \\
(0,001) *\end{array}$ & $-0,751(0,398)$ \\
\hline & $\begin{array}{l}\text { Ansiedade } \\
\text { Somática }\end{array}$ & & $0,66(0,456)$ \\
\hline \multirow{2}{*}{ Grupo 1} & $\begin{array}{c}\text { Ansiedade } \\
\text { Cognitiva }\end{array}$ & $0,304(0,072)$ & $-0,150(0,381)$ \\
\hline & $\begin{array}{c}\text { Ansiedade } \\
\text { Somática }\end{array}$ & & $-0,174(0,310)$ \\
\hline \multirow{2}{*}{ Grupo 2} & $\begin{array}{c}\text { Ansiedade } \\
\text { Cognitiva }\end{array}$ & $\begin{array}{c}0,518 \\
(0,001)^{*}\end{array}$ & $-0,186(0,276)$ \\
\hline & $\begin{array}{c}\text { Ansiedade } \\
\text { Somática }\end{array}$ & & $0,133(0,438)$ \\
\hline \multirow{2}{*}{ Grupo 3} & $\begin{array}{c}\text { Ansiedade } \\
\text { Cognitiva }\end{array}$ & $\begin{array}{c}0,494 \\
(0,001)^{*}\end{array}$ & $0,131(0,330)$ \\
\hline & $\begin{array}{l}\text { Ansiedade } \\
\text { Somática }\end{array}$ & & $0,214(0,111)$ \\
\hline
\end{tabular}

$r(p): r$ = Correlação de Pearson; $p=$ nível de significância. * Correlação moderada e alta.

Agora, separando a amostra por sexo, os resultados não demonstraram diferença entre homens e mulheres para nenhuma das subescalas: ansiedade somática $[\mathrm{t}(127)=-0,482, \mathrm{p}>0,631]$; ansiedade cognitiva $[\mathrm{t}(127)=-1,215$, $\mathrm{p}>0,227]$; autoconfiança $[\mathrm{t}(127)=1,172, \mathrm{p}>0,243]$. Tais resultados demonstram que homens e mulheres não apresentam valores similares de ansiedade e de autoconfiança, tendo a autoconfiança valores superiores aos de ansiedade somática e cognitiva.

Em relação as análises intragrupo os resultados apresentaram diferenças significantes para o sexo masculino $(p<0,05)$. O Post Hoc apresentou diferença significante entre a subescala autoconfiança e as demais $(p<0,025$, valor de $p$ ajustado), ou seja, os homens apresentam valores da subescala autoconfiança superiores aos de ansiedade. Para o sexo feminino, os mesmos valores foram encontrados, ou seja, tanto para homens, quanto para mulheres, a subescala autoconfiança apresentou-se superior à ansiedade somática e cognitiva.

Em síntese, os resultados do presente estudo demonstraram que os nadadores de águas abertas, independentemente da faixa etária ou do sexo, apresentam valores superiores para subescala autoconfiança. Além disso, na análise por idade, o grupo 3, formado por nadadores mais velhos, apresentou valores superiores de ansiedade somática, em relação aos demais grupos, mas mesmo assim é inferior ao valor da autoconfiança. Por fim, existe uma correlação forte e positiva entre a subescala somática e a cognitiva, sugerindo que uma influencia diretamente a outra, corroborando a literatura (FERNANDES et al., 
2014; FERNANDES; VASCONCELOS-RAPOSO; FERNANDES, 2012a, 2012b; VIEIRA et al., 2011).

Sobre o instrumento utilizado, alguns estudos investigaram suas propriedades psicométricas (BARTHOLOMEU; MONTIEL; MACHADO, 2013; COELHO; VASCONCELOS-RAPOSO; MAHL, 2010; FERNANDES; VASCONCELOSRAPOSO; FERNANDES, 2012a) e os mesmos demonstraram que o questionário apresenta bons resultados de validade e de fidedignidade, corroborando os resultados encontrados por Paludo et al. (2016), Fernandes, Vasconcelos-Raposo e Fernandes (2012a) e Teixeira (2016), ou seja, a versão do CSAI-2R utilizada, com 17 itens, apresenta boas propriedades psicométricas, com evidências de validade e precisão.

Todavia, retornando aos resultados, o presente estudo apresentou resultados de elevada autoconfiança e baixa ansiedade cognitiva e somática, independentemente das análises serem por grupo de idade ou de sexo. Tais achados estão em conformidade com a teoria proposta por Martens, Vealey e Burton (1990), a Teoria Multidimensional da Ansiedade e com os resultados de alguns estudos (ARRUDA et al., 2017; BERNARDT; SEHNEM, 2017; COWDEN; FULLER; ANSHEL, 2014; FERNANDES et al., 2013a; FERNANDES; VASCONCELOSRAPOSO; FERNANDES, 2012a; FORTES et al., 2017; HAGAN JR.; POLLMANN; SCHACK, 2017; NASCIMENTO JUNIOR et al., 2016; PAES et al., 2016).

De acordo com a Teoria Multidimensional da Ansiedade (MARTENS; VEALEY; BURTON, 1990), quando os atletas apresentam elevados valores de autoconfiança, os escores de ansiedade somática e cognitiva se apresentam baixos, ou seja, a autoconfiança atua como inibidor da ansiedade, ou melhor, como uma estratégia de coping que auxilia no manejo dos níveis de ansiedade. Tais achados são importantes para as intervenções junto aos atletas, pois os psicólogos do esporte, pensando-se em auxiliar o atleta frente ao controle de ansiedade, podem propor estratégias de aumento da autoconfiança, já que a mesma se apresenta como um fator que diminuiu a ansiedade. Além disso, os resultados de correlação do presente estudo entram em consonância com os princípios da teoria, já que o presente estudo demonstrou correlação forte e positiva entre a ansiedade somática e a cognitiva, sugerindo-se a influência de uma sobre a outra, conforme propõe a teoria e alguns estudos (BERNARDT; SEHNEM, 2017; FERNANDES et al., 2014; FERNANDES; VASCONCELOS-RAPOSO; FERNANDES, 2012a, 2012b; HAGAN JR.; POLLMANN; SCHACK, 2017; NASCIMENTO JUNIOR et al., 2016; VIEIRA et al., 2011).

Tratando-se das análises por idade, era esperado que a experiência tivesse influência nos resultados do estudo, ou seja, que o grupo com pessoas mais velhas demonstrasse escores de ansiedade menores e autoconfiança superiores aos grupos mais jovens. Todavia, tal hipótese não foi suportada no presente estudo, já que os resultados não demonstraram superioridade do grupo mais velho, ou 
seja, com mais experiência em competição.

Tais achados diferem da literatura quando se trata de atletas de elite (FERNANDES et al., 2014; NASCIMENTO JUNIOR et al., 2016; NOGUEIRA, 2016; PAES et al., 2016; VIEIRA et al., 2011). De acordo com a literatura (FERNANDES et al., 2013a) os atletas mais experientes interpretam os sinais de ansiedade e autoconfiança como facilitadores para o desempenho. Em contrapartida, os atletas menos experientes interpretam os sinais de ansiedade e autoconfiança como dificultadores do desempenho (FERNANDES et al., 2014).

Entretanto, como já mencionado, no presente estudo tal hipótese não foi confirmada, pois não houve diferença significantes entre os grupos de idade. Possivelmente a diferença entre os achados e os resultados da literatura deve-se pelo fato de a amostra ser amadora e não atletas de elite. De acordo com Paes et al. (2016), os atletas amadores apresentam níveis baixos de ansiedade somática e cognitiva, possivelmente pela menor cobrança que se tem sobre o atleta.

Assim, os achados do presente estudo demonstram que os atletas amadores apresentam valores mais baixos de ansiedade somática e cognitiva, corroborando com a literatura (BARRETO, 2017; BOAS et al., 2012; FERNANDES et al., 2014; PAES et al., 2016; SOUZA; TEIXEIRA; LOBATO, 2012). Tais achados reforçam, novamente, a importância do psicólogo do esporte na preparação dos atletas, principalmente frente aos atletas de alto rendimento, uma vez que cobranças externas pela vitória podem influenciar diretamente os níveis de ansiedade e, com isso os atletas podem interpretar tais informações como dificultadoras para o desempenho (FERNANDES et al., 2013a).

Em relação às análises por sexo, observou-se no presente estudo que os resultados não demonstraram diferenças entre homens e mulheres, o que difere de alguns estudos (BORREGO; CID; SILVA, 2012; FERNANDES et al., 2013a; NASCIMENTO JUNIOR et al., 2016; OZAKI; ARANTES, 2012). Porém, os resultados do presente estudo são suportados pela literatura (BERTUOL; VALENTINI, 2006; HAGAN JR.; POLLMANN; SCHACK, 2017; SEELEY et al. , 2000; VIEIRA et al., 2011), os quais não se encontrou diferenças entre homens e mulheres para nenhuma subescala do instrumento, ou seja, as mulheres demonstram níveis similares aos homens e, além disso, os 2 grupos apresentam elevados escores de autoconfiança, o que diminui os escores de ansiedade somática e cognitiva (HAGAN JR.; POLLMANN; SCHACK, 2017). Tal explicativa pode estar relacionada ao fato de que as diferenças de sexo não apresentam relevância enquanto competição, pois o atleta independentemente do sexo mantém o propósito de ter êxito em sua performance.

Tais resultados sugerem que as equipes investigadas, mesmo que amadoras, podem estar engajadas na preparação mental dos atletas, o que contribui com o manejo das emoções e aumento da autoconfiança (VIEIRA et al., 2011). Todavia, 
a questão de engajamento em preparação mental não foi foco do presente estudo, mas, mesmo assim, os resultados apontam novamente a importância do psicólogo do esporte na preparação mental dos atletas para lidarem com situações estressoras, como a ansiedade, através de propostas de aumento da autoconfiança, o que é denominado pela literatura de tenacidade mental (COWDEN; FULLER; ANSHEL, 2014). Esse resultado merece destaque, pois demonstra indícios de que a autoconfiança parece funcionar como estratégia de coping para o enfrentamento da ansiedade que a competição gera no atleta antes de seu início (NASCIMENTO JUNIOR et al., 2016).

Diante de tais hipóteses, ou seja, que a autoconfiança pode proporcionar aos atletas estratégias de enfrentamento diante de situações de competição, os resultados do presente estudo e as interpretações que a literatura aponta sobre o papel da autoconfiança para controle da ansiedade (HAGAN JR.; POLLMANN; SCHACK, 2017), remetem a ideia de Bandura (1997) na qual o atleta deve lidar com fatores estressores da competição e não comprometer seu rendimento na mesma. Além disso, a literatura sugere que atletas autoconfiantes sentem-se mais à vontade diante de situações competitivas, concentram-se melhor em seus objetivos e tarefas, facilitando o alcance de melhores desempenhos (BERNARDT; SEHNEM, 2017; TSOPANI; DALLAS; SKORDILIS, 2011).

Como todos os participantes do estudo já nadaram em outras competições, a experiência com as outras competições podem ter influenciado a interpretação dos participantes, frente as exigências da competição, o que contribui, positivamente, para o aumento da autoconfiança dos nadadores, corroborando com os achados da literatura (BERNARDT; SEHNEM, 2017; FERNANDES et al., 2014; FERNANDES; VASCONCELOS-RAPOSO; FERNANDES, 2012a, 2012b; HAGAN JR.; POLLMANN; SCHACK, 2017; NASCIMENTO JUNIOR et al., 2016; NOGUEIRA, 2016; BAUZÁ et al., 2018; VIEIRA et al., 2011).

Assim o presente tem como avanço a demonstração da importância da autoconfiança para o controle da ansiedade em nadadores de águas abertas, o que corroboram com a literatura de outras modalidades esportivas (BERNARDT; SEHNEM, 2017; CARRON et al., 2002; LUNDQVIST; KENTTÄ; RAGLIN, 2011; MODROÑO; GUILLÉN, 2011; PAES et al., 2016; BAUZÁ et al., 2018), bem como estudos que utilizaram atletas de natação de águas confinadas (BARBACENA; GRISI, 2008; FORTES et al., 2017; SOUZA; TEIXEIRA; LOBATO, 2012). Ainda é oportuno destacar, que o psicólogo do esporte tem papel importante nesse processo de competição, já que o mesmo pode propor ao atleta estratégias de aumento da autoconfiança, e demonstrando a necessidade do mesmo para preparação mental de atletas.

Ainda cabe destacar que, o presente estudo apresenta algumas limitações que merecem ser apresentadas: i) a amostra foi composta por atletas amadores, o que remete a necessidade de comparação com atletas de elite da natação de 
águas abertas (LUNDQVIST; KENTTÄ; RAGLIN, 2011), ii) os resultados do presente estudo não foram comparados com outras medidas, como por exemplo medidas fisiológicas (ARRUDA et al., 2017; FORTES et al., 2017; THOMAS; PICKNELL; HANTON, 2011), iii) a experiência dos atletas não foi investigada, enquanto influência nos níveis de ansiedade e iv) utilizou-se apenas a dimensão intensidade, demonstrando cautela para discutir os resultados de forma individual (a cada atleta) (FERNANDES et al., 2013b), já que o instrumento utilizado suportase numa abordagem nomotética (JONES et al., 1995).

\section{CONSIDERAÇÕES FINAIS}

Os resultados do presente estudo demonstraram que os nadadores de águas abertas, amadores, apresentaram um elevado escore de autoconfiança e que esse elevado escore tem direta influência nos valores baixos de ansiedade cognitiva e somática. Além disso, os resultados do presente estudo sugerem que a idade e o sexo não são fatores que alteram os níveis de ansiedade pré-competitiva.

Além disso, por meio do presente estudo é possível inferir que psicólogos do esporte têm fundamental importância na preparação mental de atletas, como os amadores, já que o mesmo pode propor ao atleta estratégias de aumento da autoconfiança, como evidenciam os resultados do presente estudo. Os dados sugerem que a autoconfiança é um bom mecanismo de controle da ansiedade somática e cognitiva e, consequentemente, pode influenciar de forma positiva no rendimento do atleta.

Assim, sugere-se que estudos futuros sejam conduzidos buscando-se sanar as limitações deste estudo, bem como avançar para entendimentos acerca dos modelos nomotéticos e ideográficos, como forma de auxiliar no entendimento da relação entre ansiedade e rendimento esportivo.

\section{REFERÊNCIAS}

ARRUDA, Ademir F. S.; AOKI, Marcelo S.; PALUDO, Ana Carolina; MOREIRA, Alexandre. Salivary steroid response and competitive anxiety in elite basketball players: Effect of opponent level. Physiology \& behavior, Zurich, v. 177, n. 1, p. 291-296, 2017. Disponível em: https://www.ncbi.nlm.nih.gov/pubmed/28527679.

BAUZÁ, Juan Pons; VILADRICH, Carme; RAMIS, Yan. Examining the big three of coping in adolescent athletes using network analysis as an alternative to factorial approach. Revista de Psicología del Deporte, Barcelona, v. 26, p. 68-74, 2017. Disponível em:

BAUZÁ, Joan Pons; VILADRICH, Carme; RAMIS, Yago; POLMAN, Remco. The Mediating Role of Coping between Competitive Anxiety and Sport Commitment in Adolescent Athletes. The Spanish journal of psychology, Madrid, v. 21, p. E7, 2018. Disponível em: 
https://www.cambridge.org/core/journals/spanish-journal-of-

psychology/article/mediating-role-of-coping-between-competitive-anxiety-and-sportcommitment-in-adolescent-athletes/C61A465EDD3829A524BD5D6B152C896D.

BANDURA, Albert. Self-efficacy: The exercise of control. New York: Freeman, 1997.

BARBACENA, Marcella Manfrin; GRISI, Roseni Nunes de Figueiredo. Nível de ansiedade pré-competitiva em atletas de natação. Conexões, Campinas, v. 6, n. 1, p. 31-39, 2008. Disponível em:

https://periodicos.sbu.unicamp.br/ojs/index.php/conexoes/article/view/8637869.

BARReTO, Patrícia Marques. Perfil de estado de humor, ansiedade-traço e ansiedadeestado em jovens ginastas. 2017. 53 f. Dissertação (Mestrado em Psicologia do Desenvolvimento e Aprendizagem). Universidade Estadual Paulista Júlio de Mesquita Filho, Bauru, 2017.

BARTHOLOMEU, Daniel; MONTIEL, José Maria; MACHADO, Afonso Antonio. Avaliação da escala likert dos itens do CSAI-2 em atletas. Interação em Psicologia, Curitiba, v. 17, n. 1, p. 79-89, 2013. Disponível em: https://revistas.ufpr.br/psicologia/article/view/26775.

BERNARDT, Andréia Maria; SEHNEM, Scheila Beatriz. Ansiedade Pré-Competitiva e autoconfiança em modalidade de esporte coletivo. Pesquisa em Psicologia: anais eletrônicos, Joaçaba, p. 37-44, 2017.

BERTUOL, Lisandra; VALENTINI, Nadia Cristina. Ansiedade competitiva de adolescentes: Gênero, Maturação, Nível de experiência e Modalidades Esportivas. Revista da Educação Física da UEM, Maringá, v. 17, n. 1, p. 65-74, 2006. Disponível em:

https://www.researchgate.net/publication/258699661 Ansiedade competitiva de adoles centes Genero maturacao nivel de experiencia e modalidades esportivas.

BIAGgIO, Angela M. B.; NATALÍCIO, Luiz; SPIELBERGER, Charles D. Desenvolvimento da forma experimental em português do Inventário de Ansiedade Traço-Estado (IDATE) de Spielberger. Arquivos Brasileiros de Psicologia Aplicada, Rio de Janeiro, v. 29, n. 3, p. 31-44, 1977. Disponível em:

http://bibliotecadigital.fgv.br/ojs/index.php/abpa/article/view/17827.

BOAS, Marcelo Silva Villas; COELHO, Ricardo Weigert; VIEIRA, Lenamar Fiorese; FONSECA, Paulo Henrique Santos; KUCZYNSKI, Kátia Maria; BOAS, Angeli Graças B. Villas. Análise do nível de ansiedade de jovens atletas da Modalidade de Futsal. Conexões, Campinas, v. 10, n. 3, p. 77-86, 2012. Disponível em: https://periodicos.sbu.unicamp.br/ojs/index.php/conexoes/article/view/8637648.

BORREGO, Carla Chicau; CID, Luis; SILVA, Carlos. Relationship between group cohesion and anxiety in soccer. Journal of Human Kinetics, Mikolowska, v. 34, n. 1, p. 119-127, 2012. Disponível em: https://www.ncbi.nlm.nih.gov/pmc/articles/PMC3590828/.

CARRON, Albert V.; COLMAN, Michelle M.; WHEELER, Jennifer; STEVENS, Diane. Cohesion and Performance in Sport: A Meta Analysis. Journal of Sport and Exercise Psychology, Bath, v. 24, n. 2, p. 168-188, 2002. Disponível em: https://journals.humankinetics.com/view/journals/jsep/24/2/article-p168.xml.

COELHO, Eduarda Maria; VASCONCELOS-RAPOSO, José; MAHL, Alvaro Cielo. Confirmatory factorial analysis of the Brazilian version of the Competitive State Anxiety Inventory-2 (CSAI-2). The Spanish journal of psychology, Madrid, v. 13, n. 1, p. 453-60, 2010. Disponível em: https://www.cambridge.org/core/journals/spanish-journal-ofpsychology/article/confirmatory-factorial-analysis-of-the-brazilian-version-of-thecompetitive-state-anxiety-inventory2-csai2/36B7AC85174867BA5FFE0EBA484D31F9. 
COWDEN, Richard G.; FULLER, Dana K.; ANSHEL, Mark H. Psychological Predictors of Mental Toughness in Elite Tennis: An Exploratory Study in Learned Resourcefulness and Competitive Trait Anxiety. Perceptual and Motor Skills, Norfolk, v. 119, n. 3, p. 661-678, 2014. Disponível em: https://www.ncbi.nlm.nih.gov/pubmed/25387038.

COX, Richard H.; MARTENS, Matthew P.; RUSSELL, William D. Measuring Anxiety in Athletics: The Revised Competitive State Anxiety Inventory-2. Journal of Sport and Exercise Psychology, Bath, v. 25, n. 4, p. 519-533, 2003. Disponível em: https://journals.humankinetics.com/view/journals/jsep/25/4/article-p519.xml.

ENGLERT, Chris; BERTRAMS, Alex. Anxiety, ego depletion, and sports performance. Journal of Sport \& Exercise Psychology, Bath, v. 34, n. 5, p. 580-99, 2012. Disponível em: https://www.ncbi.nlm.nih.gov/pubmed/23027229.

FERNANDES, M. G.; NUNES, S. A. N.; VASCONCELOS-RAPOSO, J.; FERNANDES, H. M. Factors influencing competitive anxiety in Brazilian athletes. Brazilian Journal of Kinanthropometry and Human Performance, Florianópolis, v. 15, n. 6, p. 705-715, 2013a. Disponível em: https://periodicos.ufsc.br/index.php/rbcdh/article/view/1980$\underline{0037.2013 v 15 n 6 p 705}$.

FERNANDES, Marcos Gimenes; NUNES, Sandra Adriana Neves; VASCONCELOS-RAPOSO, José; FERNANDES, Helder Miguel. Efeitos da experiência nas dimensões de intensidade, direção e frequência da ansiedade e autoconfiança competitiva: Um estudo em atletas de desportos individuais e coletivos. Motricidade, Ribeira de Pena, v. 10, n. 2, p. 81-89, 2014. Disponível em:

http://www.scielo.mec.pt/scielo.php?script=sci arttext\&pid=S1646107X2014000200009.

FERNANDES, Marcos Gimenes; NUNES, Sandra Adriana; RAPOSO, José; FERNANDES, Helder Miguel; BRUSTAD, Robert. The CSAI-2: An Examination of the Instrument's Factorial Validity and Reliability of the Intensity, Direction and Frequency Dimensions with Brazilian Athletes. Journal of Applied Sport Psychology, London, v. 25, n. 4, p. 377391, 2013b. Disponível em:

https://www.tandfonline.com/doi/full/10.1080/10413200.2012.744780.

FERNANDES, Marcos Gimenes; VASCONCELOS-RAPOSO, José; FERNANDES, Helder Miguel. Propriedades Psicométricas do CSAI-2 em Atletas Brasileiros. Psicologia: Reflexão e Crítica, Porto Alegre, v. 25, n. 4, p. 679-687, 2012a. Disponível em:

http://www.scielo.br/scielo.php?script=sci arttext\&pid=S0102-79722012000400007.

FERNANDES, Marcos Gimenes; VASCONCELOS-RAPOSO, José; FERNANDES, Helder Miguel. Relação entre orientações motivacionais, ansiedade e autoconfiança, e bem-estar subjetivo em atletas brasileiros. Motricidade, Ribeira de Pena, v. 8, n. 3, p. 4-18, 2012b. Disponível em: http://www.scielo.mec.pt/scielo.php?script=sci abstract\&pid=S1646$107 \times 2012000300002 \&$ lng =pt\&nrm=iso.

FIELD, Andy. Descobrindo a estatística usando o SPSS. São Paulo: Bookman, 2009.

FORTES, Leonardo S.; COSTA, Bruna D. V. da; PAES, Pedro P.; NASCIMENTO JÚNIOR, José Roberto A. do; FIORESE, Lenamar; FERREIRA, Maria E. C. Influence of CompetitiveAnxiety on Heart Rate Variability in Swimmers. Journal of Sports Science \& Medicine, Bursa Turkey, v. 16, n. 4, p. 498-504, 2017. Disponível em: https://www.ncbi.nlm.nih.gov/pmc/articles/PMC5721179/.

GLEITMAN, Henry; REISBERG, Daniel; GROSS, James. Psicologia. 7. ed. Porto Alegre: ArtMed, 2009. 
GREEN, Samuel B.; SALKIND, Neil J.; AKEY, Theresa M. Using SPSS for windows: analyzing and understanding data. 2. ed. New Jersey: Prentice Hall, 2000.

HAGAN JR., John E.; POLLMANN, Dietmar; SCHACK, Thomas. Interaction between gender and skill on competitive state anxiety using the time-to-event paradigm: What roles do intensity, direction, and frequency dimensions play? Frontiers in Psychology, Lausanne, v. 8, n. 692, p. 1-13, 2017. Disponível em:

https://www.ncbi.nlm.nih.gov/pmc/articles/PMC5430064/.

HOPKINS, Will G. A scale of magnitudes for effect statistics. 2002. Disponível em: http://www.sportsci.org/resource/stats/index.html. Acesso em: 12 set. 2017.

JONES, Graham. More than just a game: Research developments and issues in competitive anxiety in sport. British Journal of Psychology, [s.1.], v. 86, n. 4, p. 449478, 1995. Disponível em: https://www.ncbi.nlm.nih.gov/pubmed/8542197.

JONES, Graham; SWAIN, Austin. Intensity and direction dimensions of competitive state anxiety and relationships with competitiveness. Perceptual and motor skills, Norfolk, v. 74, p. 467-472, 1992. Disponível em: https://www.ncbi.nlm.nih.gov/pubmed/1594407.

JONES, Graham; SWAIN, Austin. Predispositions to experience debilitative and facilitative anxiety in elite and non-elite performers. The Sport Psychologist, Cardiff, v. 9, n. 2, p. 201-211, 1995. Disponível em:

https://journals.humankinetics.com/view/journals/tsp/9/2/article-p201.xml.

KEISHA, Bita Mehdipoor; YUSOF, Sarina M. D.; JOURKESH, M. The relationship between pre-competition state anxiety components and mood state sub-scales socres and the result of among college athletes through temporal patterning. International Journal of Sports Sciences, New Yor, v. 5, n. 1, p. 8-15, 2015. Disponível em: http://article.sapub.org/10.5923.j.sports.20150501.02.html.

KLINE, Rex B. Promise and pitfalls of structural equiation modeling in gift research. In: THOMPSON, Bruce; SUBOTNIK, Rena F. (Eds.). Methodologies for conducting research on giftedness. Washington: American Psychological Association, 2010. p. 147-169.

LUNDQVIST, Carolina; KENTTÄ, Goran; RAGLIN, John S. Directional anxiety responses in elite and sub-elite young athletes: intensity of anxiety symptoms matters. Scandinavian journal of medicine \& science in sports, [s.1.], v. 21, n. 6, p. 853-862, 2011. Disponível em: https://www.ncbi.nlm.nih.gov/pubmed/22126716.

MARTENS, Rainer; VEALEY, Robin S.; BURTON, Damon. Competitive anxiety in sports. Champaign: Human Kinetics, 1990.

MILAVIĆ, Boris; JURKO, Damir; GRGANTOV, Zoran. Relations of competitive state anxiety and efficacy of young volleyball players. Collegium antropologicum, Zagreb, v. 37, n. 2, p. 83-92, 2013. Disponível em:

https://www.ncbi.nlm.nih.gov/pubmed/23914493.

MODROÑO, Cristián; GUILLÉN, Félix. Anxiety Characteristics of Competitive Windsurfers: Relationships with Age, Gender, and Performance Outcomes. Journal of Sport Behavior, Mobile, v. 34, n. 3, p. 281-294, 2011. Disponível em:

https://www.researchgate.net/publication/224863630 Anxiety Characteristics of Comp etitive Windsurfers Age Gender Performance Outcome.

MOLINA, José; SANDÍN, Bonifácio; CHOROT, Paloma. Sensibilidad a la ansiedad y presión psicológica: Efectos sobre el rendimiento deportivo en adolescentes. Cuadernos de 
Psicología del deporte, Murcia, v. 14, n. 1, p. 45-54, 2014. Disponível em: http://scielo.isciii.es/scielo.php?script=sci arttext\&pid=S1578-84232014000100006.

NASCIMENTO JUNIOR, José Roberto Andrade do; BALBIM, Guilherme Moraes; VISSOCI, João Ricardo Nickening; MOREIRA, Caio Rosas; PASSOS, Patrícia Carolina Borsato; VIEIRA, Lenamar Fiorese. Análise das relações entre ansiedade estado e coesão de atletas de handebol. Revista Psicologia: Teoria e Prática, São Paulo, v. 18, n. 2, p. 89102, 2016. Disponível em:

http://pepsic.bvsalud.org/scielo.php?script=sci abstract\&pid=S1516$\underline{36872016000200007 \& \operatorname{lng}=\text { pt\&nrm=iso. }}$.

NOGUEIRA, Francine Caetano Andrade. Crença de auto eficácia e ansiedade em atletas de Voleibol. 2016. 115 f. Tese (doutorado em Psicologia) - Instituto de Ciências Humanas, Universidade Federal de Juiz de Fora, Juiz de Fora, 2016.

NOGUEIRA, Francine Caetano Andrade; BARA FILHO, Maurício Gattas; LOURENÇO, Lelio Moura. Ansiedade competitiva e atletas de rendimento: Uma revisão sistemática. In: (E. L. Ferreira, F. I. Takakura, Eds.) II CONGRESSO INTERNACIONAL DA ASSOCIAÇÃO LATINO-AMERICANA DE CIÊNCIAS DO ESPORTE 2015, Juiz de Fora. Anais... Juiz de Fora: NGIME/UFJF, 2015. p.113-117.

OZAKI, Tamires Ramos Barca; ARANTES, João Pedro. Ansiedade pré-competitiva em nadadores de águas abertas. In: XV CONGRESSO DE INICIAÇÃO CIENTÍFICA DA UNIVERSIDADE DE MOGI DAS CRUZES 2012, Mogi das Cruzes. Anais... Mogi das Cruzes: Universidade de Mogi das Cruzes, 2012. p.1-4.

PAES, Mayara Juliana; MACHADO, Thais do Amaral; BERBETZ, Sabrina Rebecca; STEFANELLO, Joice Mara Facco. Frequência, intensidade e direção da ansiedade e sua relação com a coesão grupal em uma equipe de voleibol infanto-juvenil masculina. Revista Brasileira de Psicologia do Esporte, São Paulo, v. 6, n. 3, p. 46-56, 2016. Disponível em: https://portalrevistas.ucb.br/index.php/RBPE/article/view/7276.

PALUDO, Ana Carolina; NUNES, Sandra Adriana Neves; SIMÕES, Antônio Carlos; FERNANDES, Marcos Gimenes. Relação entre ansiedade competitiva, autoconfiança e desempenho esportivo: uma revisão ampla da literatura. Psicologia Argumento, Curitiba, v. 34, n. 85, p. 156-169, 2016. Disponível em:

https://periodicos.pucpr.br/index.php/psicologiaargumento/article/view/23315.

SAMULSKI, Dietmar Martin. Psicologia do Esporte: conceitos e novas perspectivas. Barueri: Manole, 2009.

SEELEY, G.; STOREY, J.; WAGNER, D.; WALKER, C.; WATTS, K. Anxiety levels and gender differences in social volleyball players before and during competition in an Australian setting. SportPsyc Unpublished, Sydney, v. 1, p. 1-5, 2000. Disponível em:

SONOO, Christi Noriko; GOMES, Anderson Luiz; DAMASCENO, Mara Laiz; SILVA, Schelyne Ribas da; LIMANA, Mirieli Denardi. Ansiedade e desempenho: um estudo com uma equipe infantil de voleibol feminino. Motriz, Rio Claro, v. 16, n. 3, p. 629-637, 2010. Disponível em: http://www.scielo.br/scielo.php?pid=S1980$65742010000300010 \&$ script $=$ sci abstract\&tlng=pt.

SOUZA, Mário Alberto Pereira; TEIXEIRA, Robson Bonoto; LOBATO, Paulo Lanes. Manifestação da ansiedade pré-competitiva em nadadores amadores. Revista da Educação Física da UEM, Maringá, v. 23, n. 2, p. 195-203, 2012. Disponível em: http://www.scielo.br/scielo.php?script=sci arttext\&pid=S1983-30832012000200004.

SWAIN, Austin; JONES, Graham. Intensity and frequency dimensions of competitive state 
anxiety. Journal of Sport Sciences, London, v. 11, p. 533-542, 1993. Disponível em: https://www.ncbi.nlm.nih.gov/pubmed/8114179.

TEIXEIRA, Karen Cristine. Construção e busca de evidências de validade de precisão de uma medida de ansiedade para atletas. 2016. 139 f. Dissertação (Mestrado em Psicologia) - Centro de Filosofia e Ciências Humanas, Universidade Federal de Santa Catarina, Florianópolis, 2016.

THOMAS, Owen; PICKNELL, Gareth; HANTON, Sheldon. Recall agreement between actual and retrospective reports of competitive anxiety: a comparison of intensity and frequency dimensions. Journal of Sports Sciences, London, v. 29, n. 5, p. 495-508, 2011. Disponível em: https://www.tandfonline.com/doi/full/10.1080/02640414.2010.541479.

TSOPANI, Despoina; DALLAS, George; SKORDILIS, Emmanouil K. Competitive state anxiety and performance in young female rhythmic gymnasts. Perceptual and motor skills, Norfolk, v. 112, n. 2, p. 549-560, 2011. Disponível em:

https://journals.sagepub.com/doi/abs/10.2466/05.09.20.PMS.112.2.549-560.

VIEIRA, Lenamar Fiores; TEIXEIRA, Cesar Luis; VIEIRA, José Luis Lopes; OLIVEIRA FILHO, Albertino. Autoeficácia e nível de ansiedade em atletas jovens do atletismo paranaense. Revista Brasileira de Cineantropometria e Desempenho Humano, Florianópolis, v. 13, n. 3, p. 183-188, 2011. Disponível em: http://www.scielo.br/scielo.php?script=sci arttext\&pid=S1980-00372011000300004.

WEINBERG, Robert S.; GOULD, Daniel. Fundamentos da psicologia do esporte e do exercício. 6. ed. Porto Alegre: ArtMed, 2017. 